\title{
Habilidades blandas en la educación y la empresa: Mapeo Sistemático
}

Fecha de recepción : 7 de noviembre de 2019 • Fecha de aceptación: 27 de febrero de 2020 • Fecha de publicación: 10 de mayo de 2020

Marcos Antonio Espinoza Mina'

Universidad Tecnológica Ecotec

mespinoza@ecotec.edu.ec

https://orcid.org/0000-0003-1530-7243

Doris Gallegos Barzola²

Mado (mercados Asociados Y Direcciones Objetivas) S.A.

doris@ecuaportales.com

\section{Resumen}

La buena actitud, el pensamiento crítico, las habilidades comunicacionales, el manejo del tiempo, entre otras, forman parte de las habilidades blandas, las que se refieren a aspectos no únicamente cognitivos del ser humano; son aptitudes que le permiten interactuar con sus pares dentro del ambiente laboral y desenvolverse en la sociedad. El objetivo de este estudio es proporcionar una revisión de estos aspectos, los cuales son muy necesarias actualmente, tanto a nivel educativo como empresarial. Se realizó un mapeo sistemático donde se extrajeron estudios que permitieron conocer sobre los conceptos dados, si se están implementando estas habilidades en la educación actual y las más requeridas para la inserción laboral. Este ensayo muestra que, aunque se están implementando sistemas, acciones y formas para incluir de manera adecuada el desarrollo y potenciación de las habilidades blandas, las empresas buscan personas dinámicas, con adaptabilidad, empoderamiento y con capacidad de reaccionar favorablemente a las adversidades; todavía no existe evidencia de su medición y no se puede determinar si serán las adecuadas y únicas para los próximos años). 


\begin{abstract}
Good attitude, critical thinking, communication skills, time management, among others, are part of soft skills, which refer to not only cognitive aspects of the human being; they are skills that allow him to interact with his peers, within the work environment and to develop in society. The objective of this study is to provide a review of the aspects of soft skills, much needed today, both educational and business. A systematic mapping was carried out where studies were extracted that allowed us to know about the concepts given, if these skills are being implemented in current education and those most required for labor insertion. This essay shows that, although systems, actions and ways are being implemented to adequately include the development and empowerment of soft skills, since companies are looking for dynamic people, with adaptability, empowerment and the ability to react favorably to adversities. ; There is still no evidence of its measurement and it cannot be determined whether they will be adequate and unique for the coming years.
\end{abstract}

Keywords: education, professional competence, work environment, skills, business. 


\section{Introducción}

Hoy en día, en muchas entrevistas de trabajo, los postulantes se encuentran con que las grandes empresas han dejado de darle relevancia a las certificaciones de calificaciones y a las experiencias con los que cuenta un candidato para pasar a enfocarse en evaluar el desarrollo de sus habilidades blandas o transversales; las mismas que tienen que ver con la aplicación de aptitudes, conocimientos y valores adquiridos.

En el informe "El desafío del desarrollo de habilidades en América Latina", se demuestra que a pesar del notable aumento en los años de escolaridad alcanzado por los adultos en los países latinoamericanos, hay evidencia consistente del desarrollo inadecuado de competencias académicas, técnicas y socioemocionales en esta región. Estas brechas representan un cuello de botella para el crecimiento de la productividad y la capacidad de los trabajadores de América Latina, para obtener un empleo bien remunerado (Banco de Desarrollo, 2016).

La colaboración y co-creación de conocimiento es una necesidad en un entorno socio-económico como el actual, algunas empresas privadas y públicas han creado espacios colaborativos para este fin. En los centros educativos se busca lograr que los estudiantes sean conscientes de lo que piensan, sienten y viven durante las clases y su relación con el contenido; poner atención en la dimensión interpersonal, promoviendo el trabajo en equipo, creando equipos para desarrollo de proyectos, así como también, poner énfasis en la dimensión social, reflexionando en el contexto en el que actúan, empoderándolos para que asuman desafíos al servicio de su comunidad. Para ello, cada una de las actividades curriculares tiene una oportunidad valiosa para promover el desarrollo socioemocional en la medida que los estudiantes llegan a ser protagonistas de su aprendizaje, generando beneficios para su desarrollo integral.

Dado este contexto, el interés de los autores de este trabajo es plantear a nivel de revisión y discusión teórica lo referente a las habilidades blandas, una realidad que en el ámbito de la formación se encuentra todavía de forma escasa. En cuanto a estas habilidades socioemocionales no existe aún un alto grado de comprensión, y esto se podría deber, según la literatura previamente revisada, a que estas son más difíciles de medir, evaluar y desarrollar. El esfuerzo realizado en el presente documento intentó identificar las habilidades blandas que deben adquirirse en la formación educativa, y que le servirán al ser humano para conseguir un buen empleo y para la vida en general.

\section{Metodología}

Para la ejecución de este trabajo se ha recurrido a un mapeo sistemático desarrollado entre los meses de octubre de 2018 y marzo de 2019; analizando artículos de revisión bibliográfica y de investigación. La información utilizada es completamente secundaria, ya que no existe un levantamiento de información primaria.

El mapeo se realizó siguiendo las etapas propuestas por Petersen, Vakkalanka, \& Kuzniarz, (2015) study selection, analysis and presentation of data, etc. quienes definieron cinco etapas: definir 
preguntas de investigación, realizar la búsqueda literaria, seleccionar estudios, clasificar artículos, extraer y realizar la agregación de datos.

El origen de los documentos corresponde a diferentes bases de información y motores de búsqueda, como lo son: Google Scholar, Scopus, Latindex, Ebscohost y ERIC; en general, los artículos seleccionados son de carácter científico afines a la temática estudiada, por lo cual se seleccionan 15 papers para su revisión.

De un modo concreto, los objetivos sobre los que se pretendió responder la investigación fueron los siguientes:

- Conocer las características de publicación alrededor del tema (años, país, tipo de metodologías y herramientas aplicadas)

- Recopilar los principales conceptos sobre habilidades blandas, compilar los métodos que permiten una adecuada puesta en marcha de las habilidades blandas a nivel educativo, e identificar las habilidades blandas mínimas requeridas a nivel laboral y de base para la futura inserción laboral de las personas.

Las palabras clave y la cadena de búsqueda fueron: (1) "habilidades blandas" y "educación", (2) "habilidades blandas" y "laboral".

En la primera búsqueda se encontró un total de 56 artículos potencialmente útiles, pero al aplicar los criterios de tipificación (inclusión y exclusión) establecidos por el equipo investigador, finalmente 14 los cumplieron. Los criterios de tipificación fueron: publicaciones arbitradas, journals, texto completo y que contuvieran los términos mencionados anteriormente en idioma español e inglés. El registro de la información se realizó en excel para realizar el análisis descriptivo.

\section{Resultados}

El total de artículos analizados fue de 14 que cumplieron los criterios de inclusión y no presentaron criterios de exclusión. El año 2016 fue el año con mayor número de publicaciones con cinco; Ecuador y Chile son los países que más han tratado sobre este tema, con tres artículos cada país. La metodología y herramienta más utilizada en las investigaciones han sido en su mayoría de revisión; se encontraron cinco artículos y le siguen las encuestas y la investigación cuantitativa, ver Tabla 1,2 y 3. 
Tabla 1.

Resultados por año de publicación

\begin{tabular}{lll}
\hline Año de publicación & No. de artículos & Porcentaje \\
\hline 2007 & 1 & $7 \%$ \\
\hline 2008 & 1 & $7 \%$ \\
\hline 2009 & 1 & $7 \%$ \\
\hline 2015 & 2 & $14 \%$ \\
\hline 2016 & 5 & $36 \%$ \\
\hline 2017 & 1 & $7 \%$ \\
\hline 2018 & 3 & $21 \%$ \\
\hline & 14 & $100 \%$ \\
\hline
\end{tabular}

Fuente: elaboración propia

Tabla 2.

Resultados por país de publicación

\begin{tabular}{lll}
\hline País de publicación & No. de artículos & Porcentaje \\
\hline Argentina & 1 & $7 \%$ \\
\hline Chile & 3 & $21 \%$ \\
\hline Colombia & 1 & $7 \%$ \\
\hline Costa Rica & 1 & $7 \%$ \\
\hline Cuba & 1 & $7 \%$ \\
\hline Ecuador & 3 & $21 \%$ \\
\hline España & 1 & $7 \%$ \\
\hline Estados Unidos & 1 & $7 \%$ \\
\hline Perú & 1 & $7 \%$ \\
\hline Rusia & 1 & $7 \%$ \\
\hline & 14 & $100 \%$ \\
\hline
\end{tabular}

Fuente: elaboración propia

Tabla 3.

Resultados por metodologías/herramientas aplicadas en la publicación

\begin{tabular}{lll}
\hline Metodología/herramientas & No. de artículos & Porcentaje \\
\hline Análisis documental & 1 & $7 \%$ \\
\hline Cuantitativa & 2 & $14 \%$ \\
\hline Cuantitativa y cualitativa & 1 & $7 \%$ \\
\hline Cuestionario & 1 & $7 \%$ \\
\hline Encuesta & 2 & $14 \%$ \\
\hline Revisión & 5 & $36 \%$ \\
\hline Revisión y análisis & 1 & $7 \%$ \\
\hline Revisión bibliográfica & 1 & $7 \%$ \\
\hline & 14 & $100 \%$ \\
\hline
\end{tabular}

Fuente: elaboración propia 
Antes de conocer los conceptos de habilidades blandas se debe identificar a las denominadas habilidades duras, que son las que responden a todo el conocimiento académico curricular obtenido durante el proceso formativo formal; estas habilidades son las que se aprenden y entre ellas se tienen: una certificación en algún área del conocimiento, el dominio de lenguas extranjeras, la práctica en informática, las técnicas de escritura y expresión oral, el conocimiento y manejo de distintas herramientas.

Olivares (2007) indica que se tiende a estratificar de manera bidimensional las competencias, como: competencias duras y competencias blandas, en que las primeras comprometen conocimientos de especialización, y las segundas habilidades relacionadas con capacidades comportamentales y elementos socio-afectivos. Se conoce que las llamadas habilidades blandas tienen su origén en la parte derecha del cerebro (Matus \& Gutierrez, 2015). Suele decirse que cualquier competencia dura puede ser entrenada rápidamente, mientras que una blanda puede tomar años (Singer, Guzmán \& Donoso, 2009).

Según el diccionario de la Real Academia Española (2019) habilidad es: capacidad y disposición para algo. Gracia y destreza en ejecutar algo que sirve de adorno a la persona. Cada una de las cosas que una persona ejecuta con gracia y destreza. Enredo dispuesto con ingenio, disimulo y maña. Partiendo de estos conceptos, se presentan de forma más amplia, los emitidos por varios autores para describir lo que son las habilidades blandas.

Para Ortega (2016) las habilidades blandas o no cognitivas, son actitudes y prácticas que afectan cómo un individuo enfoca el aprendizaje e interactúa con el mundo que le rodea. Los investigadores y los profesionales utilizan una variedad de términos para describir estos tipos de habilidades: competencias "blandas", habilidades socioemocionales, habilidades sociales y emocionales, habilidades de carácter o rasgos de personalidad.

Las habilidades blandas son un término simple para un conjunto complejo de cualidades personales que ayudan a que un individuo sea un miembro positivo y contribuyente de cualquier organización. Las habilidades sociales incluyen cualidades personales como actitud positiva, comunicación, planificación y organización, pensamiento crítico, trabajo en equipo y más (National Soft Skills Association, 2019).

El Banco de Desarrollo (2016) considera que las habilidades socio-emocionales, -también denominadas habilidades blandas, habilidades transferibles- son un amplio conjunto de habilidades que pueden adquirirse en diversos ambientes (escuela, trabajo, hogar, voluntariado) y son relevantes para cualquier tipo de trabajo.

Por su parte, en su artículo, Ortega (2017) indica que estas son un conjunto de destrezas que permiten desempeñarse mejor en las relaciones laborales y personales. Especialistas en educación coinciden en que las habilidades técnicas se pueden enseñar mucho más fácilmente que las habilidades blandas.

Mientras que Singer et al. (2009) las define como un conjunto de habilidades no-cognitivas esenciales para aprender y desempeñarse exitosamente en el trabajo. Busca demostrar que 
entrenar en jóvenes cuatro de estas competencias que son: comunicación asertiva, adaptabilidad y flexibilidad, proactividad e iniciativa, y trabajo en equipo, produce efectos positivos inmediatos.

\section{Competencias que desarrollan las habilidades blandas}

Las habilidades blandas marcan la diferencia entre dos personas, el gozar de empatía, buenos modales, capacidad de negociación, espíritu de colaboración, puntualidad y una buena dosis de optimismo, aportan una valoración especial. Estas habilidades permiten apreciar las emociones propias y expresarlas adecuadamente, entender también las emociones de los demás; estas características ayudan a orientar el pensamiento y el comportamiento hacia metas plausibles. Muchas empresas consideran que los atributos descritos facilitan la interacción con otras personas, lo cual genera un ambiente de trabajo más grato y, a la larga, favorece un clima organizacional de entendimiento y cooperación.

Matus y Gutierrez (2015) señalan que en el grupo de las competencias que estarían más cercanas a las habilidades blandas, se encuentran la adaptabilidad al cambio, el aprendizaje autónomo; y que serían más difíciles de evaluar y desarrollar, dado que son "generadas" por los rasgos de personalidad.

Se conoce, que, la nueva concepción de competencia requiere de personas con perfiles heterogéneos, capaces de trabajar en equipos e integrar sus conocimientos para dar solución a diversidad de problemas de manera creativa donde la eficiencia también es valorada, en conjunto con acciones optimizadas e innovadoras para la resolución de problemas (Olivares, 2007). Una visión generalizada de las competencias permite decir que las habilidades blandas de alguna manera determinan la capacidad para gestionar la propia actividad y desarrollo profesional, asumir la responsabilidad, resolver tareas comunicativas relacionadas con la interacción, etc. Por lo general, incluyen competencias sociales, competencias intelectuales, competencias que determinan la organización y auto-organización de las actividades (Gruzdev, Kuznetsova, Tarkhanova \& Kazakova, 2018)evaluation and development of human resources. The main focus is on analyzing the attitude of employers towards soft skills, approved by the Ministry of Education and Science of the Russian Federation for the preparation of bachelors and masters: the significance of soft skills for employees of the enterprise (organization.

La importancia de estas destrezas se ven reflejadas en tiempos de crisis, los resultados, permanencia en el tiempo; su dificultad de adquirir, búsqueda de nuevos paradigmas, salir de la zona de confort, son un desafío profesional (Honorable Senado de la Nación Argentina, 2017)2017.

En su artículo Puga y Martínez (2008) señala que las cinco habilidades mínimas que requieren poseer los gerentes o directivos de los nuevos tiempos son: orientado al conocimiento, liderazgo, habilidad para comunicar, valores éticos y capacidad para construir equipos de trabajo.

\section{Educación y habilidades blandas}

En el mundo cambiante en el que se vive, los profesionales deben convivir y comunicarse con sus 
pares, lo que sin duda contribuye al desarrollo personal; por lo cual, las habilidades blandas son capacidades comunicativas, de trabajo en equipo, adaptabilidad, empatía, pro-actividad, autocrítica y flexibilidad.

El ser humano no nace con las habilidades descritas, sino que son comportamientos adquiridos, desde la infancia, en primera instancia los padres enseñan a sus hijos, luego en el centro de estudios medios y la universidad continúan con la gran tarea de que las personas se desarrollen, pero sin olvidar que la base se establece en el hogar. Los padres tienen un rol fundamental en la formación de sus hijos para que en el futuro puedan ser seres honorables.

La educación actual ha evolucionado en el tiempo y tomado conciencia de la importancia de estas habilidades blandas, tanto en el ámbito laboral como en el cotidiano; por ello es de interés para el desarrollo de valores transversales y alcanzar el conocimiento.

Olivares (2007) sostiene que con la actual reforma educativa se fomenta un currículum basado en competencias que habilite y posibilite el desarrollo de habilidades y destrezas como elementos subordinados al logro de dichas competencias, orientadas hacia el mundo laboral.

Para los estudiantes la escuela tiene importancia en el proceso de socialización a partir de la adquisición y desarrollo de "habilidades blandas", ya que en esta tendrían la posibilidad de adquirir aquellas convenciones de comunicación legítimas en contextos sociales institucionalizados, formales y/o culturalmente hegemónicos (Espinoza, González, Castillo, \& Neut, 2018).

Es necesario elevar la calidad de la educación, mejorar la pertinencia de los programas y generar sistemas de información sobre las habilidades disponibles es importante. Articular los sistemas educativos y de formación profesional en una concepción que haga realidad la idea de educación y desarrollo de competencias a lo largo de toda la vida. Los programas educativos y los de formación para el trabajo deben tener una base de competencias socio-emocionales (blandas) que preparen para la vida y el trabajo (Vargas \& Carzoglio, 2017).

Se debe considera que no basta con hablar de un currículo por habilidades sin conseguir una aplicación correcta a los valores, para conseguir las adecuadas prácticas educativas y dentro de la organización administrativa. Comprender que cada estudiante progresa a diferentes ritmos en el dominio de una habilidad implica saber atender oportunamente sus necesidades y la personalización de las trayectorias escolares. Este cambio en la cultura y en la práctica es, sin duda, el mayor reto del sistema educativo tradicional (Portillo, 2017).

Estas destrezas pueden enseñarse utilizando diversas estrategias metodológicas, así como, juego de roles, simulación, auto-descubrimiento, cuestionamiento, entrevistas, proyectos, aprendizaje basado en problemas, aprendizaje cooperativo e instancias de reflexión. En tanto, el desarrollo de dichas habilidades podría integrarse en el currículo de varias formas: enfoque directo, enfoque metacurricular y enfoque mixto (incluye ambos enfoques) (Vera, 2016).

Para Ortega (2017) el proceso de aprendizaje informal no está sujeto a un plan escolarizado ya que sucede con las vivencias y experiencias del suceso diario, de la influencia continua 
de los medios que estructuran su entorno, por lo tanto, la familia constituye el principal grupo social en que este tipo de aprendizaje tiene lugar, a ella debe dirigirse la mayor atención y crear las condiciones para lograr un aprendizaje informal con características propias y adecuado al ambiente individual.

Con el uso inteligente de las nuevas tecnologías, la enseñanza actual presupone que una sinergia entre lo que el niño aprende en los ambientes formales e informales, plantea múltiples preguntas cuyas respuestas no son evidentes (Ortega, Febles \& Estrada, 2016).

No se trata solamente de importar y entender nuevos conceptos en educación, sino de un cambio en la filosofía, los valores, en la práctica y en la organización educativa (Portillo, 2017). Por su parte, Tito y Serrano, (2016) manifiestan que es importante que desde las universidades se prepare a los estudiantes en estas habilidades para que éstos egresen con herramientas que agreguen una ventaja diferenciadora que les permita competir en el mercado laboral, conseguir buenos empleos, obtener buenas remuneraciones, y en general mantener un elevado sentido de bienestar consigo mismo y en distintos ámbitos de su vida. Para la enseñanza de estas habilidades es necesario modificar los contenidos de la educación, confiriendo tanta importancia a los conocimientos técnicos como a las competencias blandas.

En la Tabla 4, se presentan de forma sintetizada las acciones y formas que algunos autores muestran para desarrollar las habilidades blandas en la educación, pero no se indican cuáles son los resultados de las implementaciones debido a la ausencia de estos en los trabajos revisados.

Tabla 4.

Acciones y formas para desarrollar habilidades blandas en la educación

\begin{tabular}{|c|c|}
\hline Artículos & Acciones y formas para desarrollar las habilidades blandas en la educación. \\
\hline \multirow[t]{3}{*}{ Vera (2016) } & Enfoque directo: conjunto de cursos transversales. \\
\hline & $\begin{array}{l}\text { Enfoque metacurricular: estrategias metodológicas y evaluativas de } \\
\text { todas las asignaturas. }\end{array}$ \\
\hline & Enfoque mixto: más complejo y costoso de implementar. \\
\hline \multirow[t]{5}{*}{ Ortega et al., (2016) } & $\begin{array}{l}\text { Aprender en equipos. Combinar contenidos de las clases con aspectos } \\
\text { que son aprendidos en el entorno familiar o social. }\end{array}$ \\
\hline & $\begin{array}{l}\text { Crear mapas de conocimiento (mapas mentales) que ayudan a conocer } \\
\text { las pautas estructuradas de relación y transmisión de conocimientos } \\
\text { generales. }\end{array}$ \\
\hline & $\begin{array}{l}\text { Incorporar los conceptos de complejidad, compromiso, responsabilidad, } \\
\text { autocrecimiento de las organizaciones inteligentes, sean educativas o } \\
\text { laborales. Este ejercicio se realizará una vez al mes entre los profesores. }\end{array}$ \\
\hline & Seleccionar semanalmente la mejor experiencia narrada por los alumnos. \\
\hline & Implementar un sistema de coevaluación automatizado en el aula. \\
\hline
\end{tabular}




\begin{tabular}{ll}
\hline Portillo (2017) & Construcción de mapas de progreso sólidos sobre las habilidades. \\
& Sistemas de evaluación más formativos que sumativos. \\
& Trayectorias escolares basadas en el dominio demostrado y no en la nota. \\
& Variable del tiempo en la diversidad. \\
& Apoyos a tiempo de acuerdo con las necesidades de cada estudiante. \\
\hline Tito \& Serrano (2016) & Lectura y análisis de casos de estudio. \\
& $\begin{array}{l}\text { Modelado: incluye juegos de rol. } \\
\text { Portafolios de cuestionarios y evaluaciones. } \\
\text { Simulaciones: proporcionan oportunidades para que los estudiantes } \\
\text { experimenten situaciones similares a las que encontrarán en sus puestos } \\
\text { de trabajo y carreras. }\end{array}$ \\
\hline
\end{tabular}

Fuente: elaboración propia

Durante la revisión se encontró que los autores señalan que son los docentes los primeros en ser llamados para definir y conocer claramente cuáles son sus habilidades blandas, ya que, como indica Marrero (2018), habitualmente el docente se enfrenta a situaciones a las que debe hacer frente con opciones limitadas. Sus emociones pueden influenciar su motivación y funcionamiento cognitivo, por lo cual, estudiantes con mala conducta dentro del salón de clases pueden activar emociones en éste, que evidentemente repercuten en el proceso de enseñanza -aprendizaje. Esto equivale a decir que si el docente es autorregulado, formará también personas autorreguladas. Suele decirse que la au-torregulación es esencial en el desarrollo de las habilidades socioemocionales y que constituye el sello distintivo de las personas altamente efectivas,

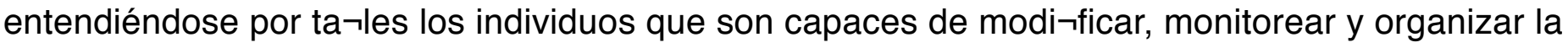
información para aplicarla a otros contextos y situa $\neg$ ciones (Vera, 2016).

\section{Empleabilidad y habilidades blandas}

Los empleadores mencionan que dentro de las dificultades para encontrar al trabajador idóneo, está la falta de profesionales integrales, capaces de tener excelentes relaciones interpersonales, con dominio de idiomas, liderazgo y competencias para el trabajo en equipo; lo que ha provocado que exista una crisis de empleabilidad. Las habilidades blandas, son muy difíciles de encontrar en un contexto donde abunda la oferta laboral, a pesar de que los sistemas de reclutamiento de ejecutivos han alcanzado un alto grado de profesionalización, inclusive si los candidatos llegan a buscar trabajo después de hacer un grado o posgrado académico. Las empresas están buscando trabajadores con mayor asertividad ante los desafíos de un mercado laboral que es dinámico, lo que implica que la persona tenga un alto nivel de adaptabilidad, orientación al logro y tolerancia a la frustración.

El 27\% de los empresarios afirman que los candidatos carecen de las habilidades técnicas o de las competencias sociales que necesitan. A nivel mundial, más de la mitad (56\%) de los empresarios indican que las habilidades de comunicación, oral y escrita, son las fortalezas humanas que más valoran, seguidas de la colaboración y la resolución de problemas (Manpower Group, 2018). 
En la Tabla 5 se muestran las habilidades blandas (soft skills) que son más se valoran a nivel empresarial.

Tabla 5.

Habilidades blandas y sus características

\begin{tabular}{ll}
\hline Habilidades blandas & Características \\
\hline Autodisciplina & $\begin{array}{l}\text { Cuando una persona se autorregula, se autogobierna, la disciplina } \\
\text { forma parte de sus habilidades blandas. }\end{array}$ \\
\hline Administración de recursos personales & $\begin{array}{l}\text { El tiempo. Hasta dónde administra y regula sus recursos directos y } \\
\text { sus prioridades. }\end{array}$ \\
\hline Habilidades de interacción con los & $\begin{array}{l}\text { Empatía o entender claramente lo que los demás necesitan de } \\
\text { demás }\end{array}$ \\
$\begin{array}{l}\text { ebservación, de diagnóstico, trabajo en equipo, además de contar } \\
\text { con inteligencia emocional para relacionarse bien con el otro; así } \\
\text { como otras competencias que, a pesar de ser más elaboradas, } \\
\text { se consideran, igualmente, habilidades blandas: Liderazgo, la } \\
\text { capacidad de inspirar a otros, de coordinar el trabajo de otros, de } \\
\text { cuidar y desarrollar a otros. }\end{array}$ \\
\hline
\end{tabular}

Fuente: elaboración propia, a partir de entrevista realizada por Sosa (2019) para Forbes

En el entorno actual, a pesar de que la tecnología; entre ellas el Internet y la redes sociales, relega la comunicación cara a cara, en el ambiente laboral hay predominio de estas habilidades blandas al momento de seleccionar personal. Tanto así, que las habilidades blandas se especifican y detallan, por ejemplo, para puestos operativos y otras para puestos ejecutivos (Sosa, 2019).

En el mercado laboral moderno el enfoque de competencias en la capacitación, la selección y la evaluación del personal, se dirige a identificar ciertas características (cualidades) de la persona, lo que brindan la capacidad para hacer algo y enfrentar con éxito las tareas y actividades individuales en general (Gruzdev et al., 2018)evaluation and development of human resources. The main focus is on analyzing the attitude of employers towards soft skills, approved by the Ministry of Education and Science of the Russian Federation for the preparation of bachelors and masters: the significance of soft skills for employees of the enterprise (organization.

En la publicación del Honorable Senado de la Nación Argentina (2017)2017, se muestra que los conocimientos técnicos o habilidades duras harán conseguir entrevistas, pero las habilidades blandas son las que harán conseguir un trabajo. Los líderes y directivos de los tiempos modernos necesitan estar renovando permanentemente sus competencias para disponer de herramientas intelectuales y prácticas de utilidad en su gestión cotidiana (Puga \& Martínez, 2008).

- Cabe mencionar que el campo de la educación superior está bajo presión como nunca antes, ya que necesita preparar a los estudiantes para una participación activa en el mundo del trabajo. Hoy los empleadores buscan profesionales que demuestren habilidades blandas -cualidades personales que los transforman en colaboradores más adaptables, más proactivos, más resilientes y más responsables (Vera, 2016). La importancia de estas habilidades en entornos educativos y laborales está creciendo rápidamente. Si bien estas habilidades son 
fáciles de notar, son difíciles de medir. Las métricas existen, pero varían de un caso a otro, y con frecuencia son más bien implícitas y vagas (Devedzic et al., 2018).

Consideran Tito \& Serrano (2016) que los empresariosde hoy, dan por sentado que se tiene suficiente preparación técnica e intelectual, para concentrarse en contratar personas que cuenten con habilidades blandas o "soft skills", y que si bien esto es considerado como una ventaja competitiva en las empresas, la comunidad educativa brinda poca importancia a la enseñanza de éstas habilidades.

\section{Organismos internacionales y habilidades blandas}

En el libro "Perspectivas económicas de América Latina 2017 juventud, competencias y emprendimiento" (OECD, 2017) se detalla que las evaluaciones de los programas de capacitación para los jóvenes muestran que combinar el aprendizaje de competencias blandas y técnicas en el aula y el puesto de trabajo con servicios de búsqueda de empleo mejora las perspectivas juveniles de lograr trabajos de calidad. Además, los países necesitan una manera eficaz de recopilar información sobre las competencias que poseen los individuos y las que necesitan las empresas. Esto ayudaría a identificar carencias y brechas, y permitiría a los países planificar las necesidades futuras, así como a volverlos más productivos y competitivos.

La Organización Internacional del Trabajo (OIT, 2017), en su documento "Desafíos de la productividad y el mundo laboral", señala la importancia de las competencias de carácter general o blandas, las que facilitan el acceso al mercado laboral al finalizar el período formativo y permiten a los actuales trabajadores adaptarse a un mercado laboral cambiante, actualizando sus competencias continuamente (aprendizaje o formación continua). Se debe verificar, la implementación de la política pública que debe ser de largo plazo, es decir, que trascienda ciclos políticos, y que proyecte sus resultados en las futuras generaciones.

Según (CAF, 2018), en la Conferencia del Banco de Desarrollo de América Latina, se indica que es imprescindible que las empresas generen una cultura mucho más ágil, abierta, menos adversa al riesgo y más tolerante al fracaso, para poder competir en el contexto empresarial actual. "Los tiempos que estamos viviendo son de grandes cambios y no van a retroceder, esto tiene implicaciones importantes en todos los niveles educativos, ya que las habilidades blandas serán las más importantes. En un mundo donde la información se encuentra de forma abundante y de todo tipo, se necesita tener criterio para saber qué información es valiosa y cuál no".

\section{Conclusiones}

Luego de hacer el mapeo sistemático y presentar los resultados encontrados, se evidencia la diferencia entre habilidades blandas y habilidades duras, siendo las habilidades duras aquellas competencias vinculadas directamente con las tareas realizadas por conocimientos y habilidades sobre un tema en específico. Por otro lado, las habilidades blandas están asociadas al comportamiento de la persona, su desempeño social, liderazgo y manejo emocional.

En dos significativos artículos se detallan las implementaciones y cambios que se han hecho en la 
educación para desarrollar las habilidades blandas, en otros se mencionan algunas características y habilidades que deben desarrollar los alumnos, tales como: componente intrapersonal, que corresponde a comprensión emocional de sí mismo, asertividad; componente interpersonal, corresponde a empatía; componentes de adaptabilidad, corresponde a solución de problemas; componentes del manejo del estrés, busca tolerancia al estrés y componente del estado de ánimo en general que corresponde a la felicidad.

En uno de los trabajos de investigación se hace énfasis en la búsqueda de elementos que permitan definir métricas para medir las habilidades blandas, ya que de momento son inconmensurables.

En la Tabla 6, se detallan los autores que tienen similitudes en las habilidades blandas encontradas y definidas por ellos, requeridas por los empleadores y expuestas en sus trabajos de investigación.

Tabla 6.

Habilidades blandas laborales más requeridas

\begin{tabular}{ll}
\hline Artículos & Habilidades blandas laborales \\
\hline Devedzic et al. (2018) & $\begin{array}{l}\text { Liderazgo, comunicación y colaboración, pensamiento crítico, } \\
\text { resolución de problemas y responsabilidad. }\end{array}$ \\
\hline Puga \& Martínez (2008) & Liderazgo, comunicación, trabajo en equipo, valores éticos. \\
\hline Honorable Senado de la Nación & $\begin{array}{l}\text { Liderazgo y manejo emocional, comportamiento personal, } \\
\text { desempeño social. }\end{array}$ \\
\hline Grgentina (2017)2017 & $\begin{array}{l}\text { Liderazgo, trabajo en equipo, comunicación, sistemas y } \\
\text { pensamiento crítico, desarrollo e implementación de proyectos. } \\
\text { Interacción intercultural, autoorganización y autoeducación, cuidado } \\
\text { de la salud, salud y seguridad }\end{array}$ \\
\hline \& Serrano (2016) & Relaciones humanas, autogestión, y eficacia en el lugar de trabajo \\
\hline
\end{tabular}

Fuente: elaboración propia

Se destaca en esta tabla que las tres habilidades blandas más requeridas por los empleadores son: liderazgo, trabajo en equipo y comunicación; luego le siguen: pensamiento crítico, resolución de problemas, responsabilidad, valores éticos, manejo emocional, comportamiento personal, desempeño social, sistemas y pensamiento crítico, desarrollo e implementación de proyectos, interacción intercultural, auto-organización y autoeducación, cuidado de la salud, salud y seguridad, relaciones humanas, autogestión, y eficacia en el lugar de trabajo.

No se evidencia en los trabajos de investigación revisados las habilidades blandas requeridas por tipo de actividad a desarrollar en el trabajador, esto a pesar de que algunos de los mismos autores exponen claramente que las habilidades necesitadas dependen del tipo de empleo ofertado; todos los artículos plantean las habilidades blandas demandadas de manera general.

Se ha demostrado que la oferta de las instituciones formadoras a nivel de enseñanza media y de educación superior técnica no se vincula de manera adecuada con la demanda del sector productivo, tanto en términos de la cantidad de trabajadores que se necesitan como en las 
competencias laborales requeridas, generando perjuicios en la empleabilidad de los jóvenes y en el desarrollo de su vida laboral. En una entrevista a Rosa María Flores Araoz Cedrón, Gerente General en Perú, en el año 2017 comenta que el crecimiento económico no ha venido acompañado con la misma intensidad de una reforma educativa orientada a desarrollar habilidades, valores y competencias necesarias para mejorar la empleabilidad, esto puede generar una desaceleración de la economía e impactar negativamente en la calidad de vida de la población en general.

Los organismos internaciones buscan coordinar sus políticas económicas y sociales para que la juventud consiga trabajos dignos, cumpliendo las expectativas de los empleadores. En sus estudios demuestran y exponen que los países se deben esforzar en fortalecer los sistemas educativos y la formación a lo largo de toda la vida, para proveer a los jóvenes de más y mejores competencias que les permitan desarrollar una mayor capacidad de adaptación al cambio y una mejor inserción y movilidad laboral.

El mapeo sistemático realizado permite extraer algunas conclusiones que se sintetizan del siguiente modo:

1. El análisis de las diferentes investigaciones ayudó a identificar los conceptos dados por los autores de los artículos revisados, en los cuales se encuentran muchas similitudes en cuanto a lo que son las habilidades blandas, ya que las describen como cualidades, actitudes y destrezas.

2. En cuanto a las competencias que se desarrollan al aplicar las habilidades blandas por lo manifestado por los autores se presentan tanto a nivel educativo como laboral, ya que, desde la etapa escolar se busca la iniciativa y la imaginación de las personas, con las actividades que se realizan en las aulas educativas. Sin embargo, se encontró más información a nivel educativo.

3. Los estudios revisados ponen de relieve que se está trabajando fuertemente sobre este tema en el campo educativo, desde la época escolar hasta la etapa universitaria; a pesar de esto, aún falta mucho por definir, por conocer claramente cuáles serán las habilidades blandas necesarias para dentro de algunos años, debido a los cambios constantes en la sociedad, tanto a nivel educativo como laboral.

4. En los artículos revisados no se encontró ningún modelo o método específico propuesto para el desarrollo de las habilidades blandas en la educación, solamente se mencionan acciones y formas, tales como: aprender en equipos, crear mapas de conocimiento (mapas mentales), incorporar los conceptos de las organizaciones inteligentes, seleccionar semanalmente la mejor experiencia narrada por los alumnos, implementar un sistema de coevaluación automatizado en el aula.

5. Con relación a la empleabilidad se conoce que las organizaciones buscan a personas con habilidades blandas definidas, entre las más requeridas están: liderazgo, trabajo en equipo y la comunicación, pero no se especifican cuáles serán las necesarias para cada tipo de empleo; inclusive no se han podido definir las métricas para cada una de ellas. 
6. Los organismos internacionales muestran muchas cifras del nivel de empleo en el que se indica que los empleadores buscan personal con habilidades blandas, pero tampoco se especifican de qué tipo y para qué tipo de empleo.

Para el futuro, se hace evidente la necesidad de continuar y profundizar la investigación en torno a esta temática y, de este modo detectar las habilidades blandas más requeridas a nivel laboral y por tipo de empleo. 


\section{Referencias bibliográficas}

Banco de Desarrollo. (2016). El desafío del desarrollo de habilidades en América Latina.

CAF. (2018). Conferencia CAF. https://www.caf.com/es/actualidad/noticias/2018/09/aumento-de-la-productividad-y-estabilidad-fiscal-las-grandes-asignaturas-pendientes-de-america-latina/

Devedzic, V., Tomic, B., Jovanovic, J., Kelly, M., Milikic, N., Dimitrijevic, S., Djuric, D., \& Sevarac, Z. (2018). Metrics for Students' Soft Skills. Applied Measurement in Education, 31(4), 283-296. https://doi.org/10.1080/ $\underline{08957347.2018 .1495212}$

Espinoza, O., González, L. E., Castillo, D., \& Neut, S. (2018). Expectativas educacionales de estudiantes que concurren a escuelas de "segunda oportunidad". 24.

Esquén, S. (2017). Entrevista "Adquirir habilidades blandas influye en el éxito laboral" Rosa María Flores Araoz Cedrón. UNICEF. https://www.unicef.org/peru/spanish/Entrevista-Rosa-Maria-Flores-Araoz-Adquirir-habilidades-blandas-influye-en-exito-laboral.pdf

Gruzdev, M., Kuznetsova, I., Tarkhanova, I., \& Kazakova, E. (2018). University Graduates' Soft Skills: The Employers' Opinion. European Journal of Contemporary Education, 7(4). https://doi.org/10.13187/ejced.2018.4.690

Honorable Senado de la Nación Argentina. (2017). Comisión de Economías Regionales, Economía Social, Micro, Pequeña y Mediana Empresa.

Manpower Group. (2018). Solucionar la Escasez de Talento Crear, Atraer, Compartir y Tranformar. Escasez de Talento.

Marrero, O. (2018). Habilidades blandas: Necesarias para la formación integral del estudiante universitario. Revista Científica Ecociencia, 18.

Matus, O., \& Gutierrez, A. (2015). Habilidades Blandas: Una ventaja competitiva en la formación tecnológica. 9.

National Soft Skills Association. (2019). The National Soft Skills Association. https://www.nationalsoftskills.org/

OECD (Ed.). (2017). Juventud, competencias y emprendimiento. OECD Publishing.

OIT. (2017). Desafíos de la productividad y el mundo laboral.

Olivares, A. M. (2007). Competencias para un mundo cognoscente. 13.

Ortega, C. (2017). Desarrollo de habilidades blandas desde edades tempranas. 89. 
Ortega, C., Febles, J., \& Estrada, V. (2016). Una estrategia para la formación de competencias blandas desde edades tempranas. Revista Cubana de Educación Superior, 35-41.

Ortega, T. (2016). Desenredando la conversación sobre habilidades blandas. 28.

Petersen, K., Vakkalanka, S., \& Kuzniarz, L. (2015). Guidelines for conducting systematic mapping studies in software engineering: An update. Information and Software Technology, 64, 1-18. https://doi.org/10.1016/j. infsof.2015.03.007

Portillo, M. C. (2017). Educación por habilidades: Perspectivas y retos para el sistema educativo. Revista Educación, 41(2), 1. https://doi.org/10.15517/revedu.v41i2.21719

Puga, J., \& Martínez, L. (2008). Competencias Directivas En Escenarios Globales. Estudios Gerenciales, 24(109), 87-103. https://doi.org/10.1016/S0123-5923(08)70054-8

Real Academia Española. (2019). Diccionario. https://dle.rae.es/?w=diccionario

Singer, M., Guzmán, R., \& Donoso, P. (2009). Entrenando Competencias Blandas en Jóvenes. 20.

Sosa, R. (2019). Aprender Soft Skills, las habilidades del futuro. https://www.forbes.com.mx/aprender-soft-skills-las-habilidades-del-futuro/

Tito, M., \& Serrano, B. (2016). Desarrollo de soft skills una alternativa a la escasez de talento humano. Innova Research Journal, 1(12), 59-76. https://doi.org/10.33890/innova.v1.n12.2016.81

Vargas, F., \& Carzoglio, L. (2017). La brecha de habilidades para el trabajo en América Latina: Revisión y análisis en la región. 28.

Vera, F. (2016). Infusión de habilidades blandas en el Currículo de la educación superior: Clave para el desarrollo de capital humano avanzado. Revista Akademeiar, 7(7), 53-73. 
Copyright (c) 2020 Marcos Antonio Espinoza Mina y Doris Gallegos Barzola

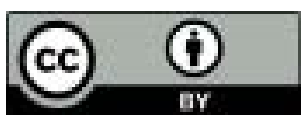

Este texto está protegido bajo una licencia internacional Creative Commons 4.0.

Usted es libre para Compartir-copiar y redistribuir el material en cualquier medio o formato - y Adaptar el documento - remezclar, transformar y crear a partir del material-para cualquier propósito, incluso para fines comerciales, siempre que cumpla las condiciones de Atribución. Usted debe dar crédito a la obra original de manera adecuada, proporcionar un enlace a la licencia, e indicar si se han realizado cambios. Puede hacerlo en cualquier forma razonable, pero no de forma tal que sugiera que tiene el apoyo del licenciante o lo recibe por el uso que hace de la obra.

$\underline{\text { Resumen de licencia - Texto completo de la licencia }}$ 